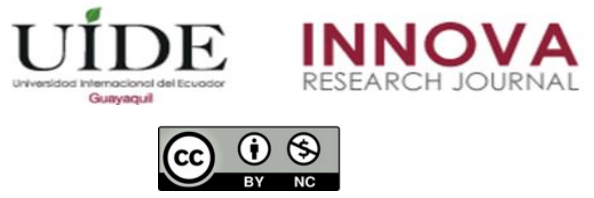

INNOVA Research Journal, ISSN 2477-9024

(Septiembre-Diciembre 2019). Vol. 4, No.3.2 pp. 1- 14

DOI: https://doi.org/10.33890/innova.v4.n3.2.2019.1138

URL: http://revistas.uide.edu.ec/index.php/innova/index

Correo: innova@uide.edu.ec

\title{
La hipótesis del ingreso permanente y la función de consumo de Ecuador. Evidencia para el periodo 2000 - 2018
}

\section{Permanent income hypothesis and the consumption function to Ecuador. Evidence for the period 2000-2018}

Javier Alejandro Solano

https://orcid.org/0000-0002-1419-8359

Valeria Elizabeth Banderas Benítez

https://orcid.org/0000-0002-6467-4958

Universidad Metropolitana, Ecuador

Autor por correspondencia: vbanderas@umet.edu.ec; jsolano@umet.edu.ec

Fecha de recepción: 10 de agosto del 2019 - Fecha de aceptación: 18 de noviembre del 2019

\section{Resumen}

Este estudio tiene como objetivo estimar una función de consumo privado agregado para la economía de Ecuador. Para ello, se contrasta la relevancia de la hipótesis de ingreso permanente de Friedman (1957) como descripción aproximada del consumo del país. Con la finalidad de comprobar empíricamente, se utilizó información trimestral durante el período 2000: q1-2018:q4, misma que fue modelada en una estimación de dos regresiones lineales basadas principalmente en el modelo racionalizado de Koyck (Gujarati y Porter, 2010), utilizando Mínimos Cuadrados Ordinarios (MCO) y Mínimos Cuadrados Ordinarios en 2 Etapas (MC2E). Los resultados sugieren que el consumo agregado en Ecuador se ajusta al nivel de ingreso real con un rezago considerable. Lo que permite evidenciar a favor de la hipótesis del ingreso permanente, en el sentido de que el gasto de consumo de los hogares responde más a los cambios en el ingreso permanente que al ingreso transitorio o del período corriente.

Palabras claves: consumo; hipótesis del ingreso permanente; modelo de Koyck

\begin{abstract}
The main objective of the present study is to estimate an aggregate private consumption function for the Ecuador's economy. For this, the relevance of Friedman's permanent income hypothesis (1957) is contrasted as an approximate description of the country's consumption. In order to verify empirically, quarterly information was used during the period 2000: q1-2018: q4, which was modeled on an estimate of two linear regressions based mainly on the streamlined Koyck model (Gujarati and Porter, 2010), using Ordinary Least Squares (OLS) and Ordinary Least Squares in 2 Stages (OLS2S). The results suggest that aggregate consumption in Ecuador is adjusted to the level of real income with a considerable lag. It allows to evidence in favor of the hypothesis of permanent income, in the sense that household consumption expenditure responds more to changes in permanent income than in transitory income or the current period.
\end{abstract}

Key words: consumption; permanent income hypothesis; Koyck's model 


\section{Introducción}

El gasto de consumo final de los hogares es uno de los mayores componentes de la demanda agregada de las economías mundiales (De Gregorio, 2007), entender su comportamiento y sus determinantes es fundamental para analizar las fluctuaciones económicas en el corto plazo y los niveles de vida a largo plazo de los países en el mundo. Adicionalmente, comprender los principales determinantes del consumo resulta beneficioso para los hacedores de política pública, específicamente de política económica, puesto que permite distinguir la influencia de fuerzas tales como el gasto público, la tecnología y la política monetaria sobre el producto agregado. Estas fuerzas influyen a su vez en el consumo de los hogares.

Existen diferentes hipótesis o teorías del consumo de los hogares, desde la teoría tradicional o microeconómica de los determinantes del consumo de los hogares, pasando por la teoría del consumo de Keynes (1936), la teoría del ciclo vital de Modigliani y Brumberg's (1954), la hipótesis del ingreso permanente de Friedman (1957) y la hipótesis del paseo aleatorio (Romer, 2006). Todas ellas describen y explican, en términos generales, factores trascendentales sobre el comportamiento y los determinantes del consumo agregado.

La principal motivación para esta investigación es analizar qué tan notables son los postulados de la hipótesis del ingreso permanente de Friedman (1957), como una posible explicación o aproximación del comportamiento del consumo de la economía ecuatoriana durante el período 2000:q1-2018:q4. Para ello, se utiliza la información disponible en el Banco Central del Ecuador -BCE- durante el período de estudio. Además, se usan dos modelos de regresión lineal múltiple: Mínimos Cuadrados Ordinarios (MCO) y Mínimos Cuadrados en 2 Etapas (MC2E) para dar mayor robustez a los resultados obtenidos.

El documento se divide en seis secciones. Luego de esta introducción, se presenta el marco analítico del estudio que abarca consideraciones de índole teórica, práctica y econométrica referente a la teoría del ingreso permanente (TIP); posteriormente, se presenta la metodología aplicada, inmediatamente se muestra los resultados y las estimaciones econométricas del estudio. Finalmente, se señalan conclusiones de la investigación y las referencias bibliográficas.

\section{Marco teórico}

La hipótesis del ingreso permanente (HIP), de Milton Friedman (1957), es la obra que en parte lo forjó como ganador del Premio Nobel de Economía en 1976. Este aporte se considera el más influyente en la comprensión de la conducta del consumidor hasta el día de hoy (Argandoña, 1990; Aguiar y Pence, 2014; Liquitaya, 2014). Friedman complementó el trabajo previo de Modigliani con su hipótesis del ciclo vital, ambos autores tomaron de base la teoría del consumidor de Fisher (1930) para afirmar que el consumo no depende solamente del ingreso actual (Duesenberry, 1967; Mankiw, 2014).

Friedman en su teoría argumentó que las decisiones de consumo de los individuos y familias están determinadas en su mayoría por sus expectativas de largo plazo. De manera específica, identificó dos componentes: uno permanente y otro transitorio, presentes tanto en el consumo como en el ingreso. Además, encontró que las relaciones de proporcionalidad se 
evidencian únicamente en la parte permanente, y están determinadas por la razón entre riqueza humano y no humano de los individuos (De Gregorio, 2007).

Esta teoría ha sido poco aplicada en los países de Latinoamérica (Liquitaya, 2014). Por ejemplo, se realizó una comparación internacional de las funciones de consumo de 18 países considerando datos entre 1950 y 1959. Los países considerados para el análisis oscilan entre países desarrollados como, por ejemplo, Estados Unidos, Canadá, Nueva Zelanda y países subdesarrollados como Honduras, Ecuador y la República del Congo. Este estudio concluye que el consumo de estos países está determinado por los niveles de ingresos, ratios de crecimiento, nivel de industrialización e incentivos a la inversión (Yang, 1964). Adicionalmente, existe un análisis de 19 países latinoamericanos acerca de la teoría del ingreso permanente entre los años 1990 y 2010 que utiliza una regresión por mínimos cuadrados ordinarios. Su principal resultado está relacionado con la elasticidad del consumo en el corto plazo pues se muestra menor que en el largo plazo con respecto al ingreso permanente (Liquitaya, 2014).

En el caso ecuatoriano, se investigó el ingreso permanente mediante los trimestres de los años 2000 al 2014 para evaluar el poder explicativo de las variables de consumo, ingreso y tasa de interés a través del método de mínimos cuadrados ordinarios. Los resultados de este estudio recalcan la carencia de significancia estadística de las variables en mención en la economía ecuatoriana (Bonilla et al., 2018).

De acuerdo a esta revisión, la presente investigación incorpora elementos innovadores como la regresión bietápica como principal instrumento; así como, la inclusión del modelo de Koyck. Además, incorpora una actualización de la información disponible en el Banco Central del Ecuador pues, se consideran los trimestres entre los años 2000 y 2018. Adicionalmente, como los artículos citados anteriormente, se realizan algunas pruebas para aseverar la significancia del modelo utilizado.

\section{Hipótesis del modelo}

Friedman (1957) en la TIP establece que el consumidor ajusta su gasto a las variaciones en sus expectativas de ingresos a largo plazo, eludiendo las variaciones transitorias de su ingreso corriente. El modelo que sostiene la TIP, incluye una relación funcional, dos definiciones y un supuesto que puntualiza la correlación de las variables consideradas:

$$
\begin{gathered}
c^{p}=\kappa(r, \omega, \eta) \cdot y^{p} \\
y=y^{p}+y^{t} \\
c=c^{p}+c^{t} \\
\rho\left(y^{p}, y^{t}\right)=\rho\left(c^{p}, c^{t}\right)=\rho\left(c^{t}, y^{t}\right)=0
\end{gathered}
$$

Donde el consumo y el ingreso observado están representados con “c $c$ ” “ $y$ ”, respectivamente; en tanto, los superíndices $p$ y $t$ identifican al componente permanente y transitorio de forma respectiva. La ecuación [1] muestra que el consumo permanente es una proporción " $\kappa$ " del ingreso permanente ${ }^{1}$; determinando que el cociente entre ambos es independiente del tamaño del ingreso permanente, aunque es dependiente de la tasa de interés

\footnotetext{
${ }^{1}$ Renta o ingreso promedio esperada en el largo plazo de la renta laboral esperada (riqueza humana). 
" $r$ " a la que el consumidor puede prestar o pedir prestado; además de la relativa importancia del ingreso de la propiedad y no propiedad, representado por el cociente de la riqueza no humana a ingreso " $\omega$ ", y de los factores identificados por la variable portmanteau " $\eta$ ", compuesta de gustos y preferencias de consumo versus las adiciones a la riqueza.

Las ecuaciones [2] y [3] especifican el vínculo entre los componentes permanentes y las magnitudes de medida, determinando que la renta registrada durante un período de tiempo es la suma del componente del ingreso permanente y el ingreso transitorio; además, dado que el consumo permanente depende del ingreso permanente, en términos prácticos el ingreso permanente debe ser una cantidad que el consumidor establece como determinante de su consumo planeado.

La última ecuación [4] plantea el supuesto de no correlación entre los componentes del ingreso y consumo permanente con su contraparte transitoria, fijando una única relación entre los componentes permanentes, este supuesto es útil para dar carácter predictivo al modelo.

\section{Interpretación de las funciones estimadas del consumo: casos de estudios empíricos}

La teoría tradicional de la función keynesiana del consumo postula que el consumo depende del ingreso corriente disponible. Keynes (1936) sostenía que «el volumen de consumo agregado depende principalmente del volumen o nivel de ingreso agregado» y que esta relación es «una función bastante estable». Más adelante, Keynes añade que es «también obvio que un nivel de ingreso más alto en términos absolutos conducirá, por lo general, a una proporción mayor de ahorro» (Keynes, 1936).

La importancia de la función de consumo en el análisis keynesiano de las fluctuaciones económicas, en el corto plazo, ha llevado a muchos investigadores a tratar de estimar la relación entre el gasto de consumo y el ingreso corriente. En contra de los postulados de Keynes, estos estudios no han demostrado, en términos generales, una relación sistemática estable. Para las economías domésticas y en un período de tiempo concreto, la relación es efectivamente de naturaleza keynesiana. Pero en un país y a lo largo del tiempo, el consumo agregado es básicamente proporcional al ingreso agregado. Adicionalmente, la función de consumo puede variar si se realiza un corte transversal entre los diferentes grupos de la población: por ejemplo, existen estimaciones que sugieren que la pendiente de la función estimada de consumo es similar para las personas de color de piel blanca y negra, pero el punto de intersección es más alto en el caso de los blancos (Romer, 2006).

Como señala Friedman (1957), la hipótesis o la TIP permite explicar claramente estos resultados. Supongamos, que el consumo viene efectivamente determinado por el ingreso permanente: $c=y^{p}$. El ingreso corriente es la suma del ingreso permanente y transitorio tal como lo expresa la ecuación [2]. Y puesto que el ingreso transitorio refleja las desviaciones del ingreso corriente con respecto al permanente, en la mayoría de las muestras su valor medio es aproximadamente cero (0) y básicamente no aparece relacionada con el ingreso permanente (Romer, 2006).

\footnotetext{
${ }^{2}$ Ganancias esperadas de la tenencia de activos financieros y físicos (riqueza no humana).

${ }^{3}$ Se traduce como "baúl de viaje", término derivado de que $\eta$ está compuesta de varios factores. 
Al considerar una regresión del consumo sobre el ingreso corriente, se tiene que:

$$
c_{i}=a+b y_{i}+e_{i}[5]
$$

En una regresión de una sola variable, el coeficiente estimado de la variable independiente es el cociente entre la covarianza de las variables independiente y dependiente y la varianza de la variable independiente. En el caso que se está analizando, siguiendo a Romer (2006), esto implica:

$$
\hat{b}=\frac{\operatorname{Cov}(y, c)}{\operatorname{Var}(y)}=\frac{\operatorname{Cov}\left(y^{p}+y^{t}, y^{p}\right)}{\operatorname{Var}\left(y^{p}+y^{t}\right)}=\frac{\operatorname{Var}(y)}{\operatorname{Var}\left(y^{p}\right)+\operatorname{Var}\left(y^{t}\right)}
$$

Aquí, la segunda igualdad refleja el hecho de que el ingreso corriente es igual al ingreso permanente más el ingreso transitorio y de que el consumo es igual al ingreso permanente y la última igualdad recoge el supuesto de que el ingreso permanente y el ingreso temporal no están correlacionados. Además, la constante estimada es igual al valor medio de la variable dependiente menos el coeficiente estimado de la pendiente multiplicado por la media de la variable independiente. Por lo tanto:

$$
\hat{a}=\bar{c}-\hat{b} \bar{y}=\bar{y}^{p}-\hat{b}\left(y^{p}+y^{t}\right)=(1-\hat{b}) \bar{y}^{p}
$$

Donde esta última igualdad se sirve del supuesto de que la media del ingreso transitorio es igual a cero (0) (Romer, 2006).

Por consiguiente, la TIP predice que la clave para determinar la pendiente de una función de consumo estimada, $\hat{b}$, es la variación relativa del ingreso permanente y del transitorio. Dicho de forma intuitiva, un incremento en el ingreso corriente irá asociado a un aumento del consumo sólo en la medida en que también refleje un incremento en el ingreso permanente. Cuando el cambio en el ingreso permanente es mucho mayor que el cambio en el ingreso transitorio, casi todas las diferencias en el ingreso corriente reflejan diferencias en el ingreso permanente y, por tanto, el consumo aumenta prácticamente en una proporción de uno a uno con el ingreso corriente. Pero cuando el cambio en el ingreso permanente es pequeño en relación con el experimentado por el ingreso transitorio, el aumento del ingreso corriente tiene poco que ver con el ingreso permanente y el consumo crecerá poco, en términos relativos (Romer, 2006).

Este análisis se puede utilizar para comprender las funciones estimadas de consumo en la literatura empírica. En el caso de los hogares, una buena parte de la variación en el ingreso refleja factores como el desempleo o el hecho de que los hogares se encuentran en diferentes momentos de su ciclo vital. En consecuencia, el coeficiente estimado de la pendiente es sustancialmente inferior a la unidad y la intersección estimada es positiva. Por el contrario, casi toda la variación del ingreso agregado que tiene lugar en el tiempo es un reflejo del crecimiento a largo plazo, esto es, de un aumento de los recursos de que dispone la economía; de ahí que la pendiente estimada se acerque a la unidad y la intersección estimada esté próxima a cero (0). Considerando el caso de las diferencias de las estimaciones de las funciones de consumo entre negros y blancos. La variación relativa del ingreso permanente y transitorio son similares en los 
dos grupos y de ahí que la estimación de $b$ sea similar en ambos. Sin embargo, el ingreso medio de los negros es inferior al de los blancos, de modo que $a$ es menor en el caso del primer grupo, por ejemplo (Romer, 2006).

\section{Racionalización del modelo de Koyck: el modelo de expectativas adaptativas}

El modelo de Koyck (1954) surge como un método ingenioso de estimación de los modelos de rezagos distribuidos, aunque es un modelo ad hoc puesto que su obtención se basa en un proceso puramente algebraico, desprovisto originalmente de un soporte teórico, se han desarrollado diversas aplicaciones relacionadas con los modelos de expectativas adaptativas y con el modelo de ajuste parcial de existencias ${ }^{4}$. Para ejemplificar o ilustrar el método de racionalización del modelo de Koyck, siguiendo a Gujarati y Porter (2010), se parte del siguiente modelo de rezago distribuido infinito:

$$
y_{t}=\alpha+\beta_{0} x_{t}+\beta_{1} x_{t-1}+\beta_{1} x_{t-2}+\cdots+u_{t}
$$

Si se supone que las $\beta$ tienen todos los mismos signos, Koyck da por hecho que éstos se reducen geométricamente de la siguiente manera:

$$
\beta_{k}=\beta_{0} \lambda^{k} \quad k=0,1 \ldots
$$

Donde $\lambda$, tal que $0<\lambda \leq 1$, se conoce como la tasa de descenso, o de caída, del rezago distribuido y donde $1-\lambda$ se conoce como la velocidad de ajuste.

Lo que se postula en [9] es que cada coeficiente $\beta$ anterior (esta afirmación se debe a que $\lambda<1$ ), lo cual implica que a medida que se retorna al pasado distante, el efecto de ese rezago sobre $y_{t}$ se hace progresivamente menor, un supuesto bastante razonable. Después de todo, se espera que los ingresos actuales y del pasado reciente afecten al gasto de consumo actual con mayor peso que el ingreso en el pasado distante (Gujarati y Porter, 2010).

El valor del coeficiente rezagado $\beta_{k}$, depende, a parte del $\beta_{0}$ común, del valor de $\lambda$. Entre más cerca de 1 esté $\lambda$, más lenta será la tasa de descenso en $\beta_{k}$, mientras que, entre más cerca de cero, más rápido será el descenso en $\beta_{k}$. En el primer caso, los valores del pasado distante de $x$ ejercerán un impacto considerable sobre $y_{t}$, mientras que en el último caso, su influencia sobre $y_{t}$ desaparecerá rápidamente (Gujarati y Porter, 2010). Entre las características del esquema de Koyck se encuentran: 1) al suponer valores no negativos para $\lambda$, Koyck elimina la posibilidad de que las $\beta$ cambien de signo; 2) al suponer que $\lambda<1$, le da un menor peso a las $\beta$ en el pasado distante que a las actuales, y 3 ) asegura que la suma de las $\beta$, que proporciona el multiplicador de largo plazo, es finita, a saber:

$$
\sum_{k=0}^{\infty} \beta_{k}=\beta_{0}\left(\frac{1}{1-\lambda}\right)
$$

\footnotetext{
${ }^{4}$ Para mayores detalles sobre la aplicación del modelo o método de Koyck para los modelos de expectativas adaptativas y de ajuste parcial de existencias, así como también una combinación de ambos enfoques véase a: Gujarati y Porter (2010) y Greene (1999). 
Como resultado de [9], el modelo de rezagos infinitos [8] se puede escribir como:

$$
y_{t}=\alpha+\beta_{0} x_{t}+\beta_{0} \lambda x_{t-1}+\beta_{0} \lambda^{2} x_{t-2}+\cdots+u_{t}
$$

Tal como está planteado, el modelo aún no está adecuado para su fácil estimación puesto que un gran número (literalmente infinito) de parámetros queda aún por ser estimado y el parámetro $\lambda$ ingresa en una forma por completo no lineal; estrictamente hablando, el método de análisis de regresión lineal (en parámetros) no puede ser aplicado a un modelo de este tipo (Gujarati y Porter, 2010). No obstante, Koyck sugiere una forma ingeniosa para lograrlo. Rezaga la ecuación [11] en un período para obtener:

$$
y_{t-1}=\alpha+\beta_{0} x_{t-1}+\beta_{0} \lambda x_{t-2}+\beta_{0} \lambda^{2} x_{t-3}+\cdots+u_{t-1}
$$

Luego multiplica [12] por $\lambda$ para obtener:

$$
\lambda y_{t-1}=\lambda \alpha+\lambda \beta_{0} x_{t-1}+\beta_{0} \lambda^{2} x_{t-2}+\beta_{0} \lambda^{3} x_{t-3}+\cdots+\lambda u_{t-1}
$$

Al restar [13] de [11] se obtiene:

$$
y_{t}-\lambda y_{t-1}=\alpha(1-\lambda)+\beta_{0} x_{t}+\left(u_{t}-\lambda u_{t-1}\right)
$$

Reordenando,

$$
y_{t}=\alpha(1-\lambda)+\beta_{0} x_{t}+\lambda y_{t-1}+v_{t}
$$

Donde $v_{t}=\left(u_{t}-\lambda u_{t-1}\right)$ es un promedio móvil de $u_{t}$ y $u_{t-1}$.

El procedimiento recién descrito se conoce como la transformación de Koyck. Comparando [15] con [8], se puede apreciar la enorme simplificación lograda por Koyck. Mientras antes era preciso estimar $\alpha$ y un número infinito de $\beta$, ahora se tienen que estimar solamente tres incógnitas: $\alpha, \beta$ y $\lambda$. Ahora bien, no hay razón para esperar multicolinealidad. En cierto sentido, la multicolinealidad se resuelve reemplazando $x_{t-1}, x_{t-2}, \ldots$, por una variable única, a saber $y_{t-1}{ }^{5}$.

\section{Metodología}

En este estudio se persigue estimar una función de consumo trimestral para la economía de Ecuador durante el período (2000:q1-2015:q3) siguiendo, por una parte, los postulados y las implicaciones de la TIP (Friedman, 1957) sobre la base de la aplicación de la racionalización del modelo de Koyck (1954) para los efectos de las estimaciones econométricas a través de Mínimos Cuadrados Ordinarios (MCO) y el método de Variable Instrumental (VI), específicamente, el método de Mínimos Cuadrados en 2 Etapas (MC2E), con el propósito de contrastar, en cierta forma, si el gasto de consumo final de los hogares en Ecuador durante el período de estudio se

\footnotetext{
${ }^{5}$ Para mayores detalles sobre el método de Koyck y los cálculos implícitos y explícitos (como la mediana de los rezagos y el rezago medio) a partir de los parámetros véase a: Gujarati y Porter (2010) y Greene (1999).
} 
aproxima o no a la hipótesis del ingreso permanente de Friedman (1957). La razón principal para aplicar el método de MC2E facilita el cumplimiento de la eficiencia de los estimadores en el modelo.

\section{Datos y análisis descriptivo de las variables}

En el presente estudio se utilizan datos trimestrales sobre el gasto de consumo final real de los hogares (GC), el nivel de ingreso real, aproximado por el producto interno bruto (Y) y la tasa de interés pasiva del sistema bancario en términos porcentuales (I), para representar el costo de oportunidad del dinero y del consumo presente (ahorro), los datos considerados corresponden al periodo comprendido entre el 2000 y el 2018. Esta información se obtuvo de las bases de datos oficiales del Banco Central del Ecuador (BCE, 2018). En este sentido, en la figura 1 se presenta la evolución temporal de estas tres variables en sus niveles logarítmicos (primera fila), en primeras diferencias (segunda fila) y en tasas de variación trimestrales anualizadas (tercera fila).
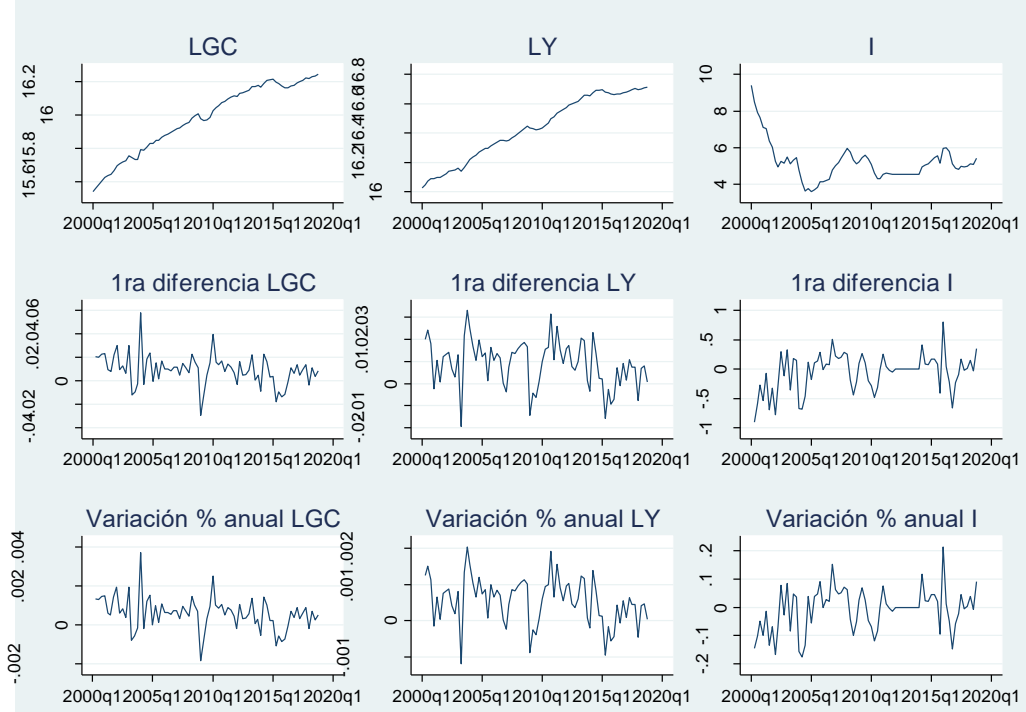

Figura 1. Evolución de las variables del estudio. Período (2000:q1-2018:q4).

Fuente: BCE y elaboración propia

Por otra parte en la tabla 1 se presenta, como análisis previo de las estimaciones econométricas, el resumen de las estadísticas descriptivas de las variables del estudio, en donde se destaca que según la prueba de normalidad Jarque-Bera (JB) el logaritmo del gasto de consumo real (GC) no sigue una distribución normal mientras, el logaritmo del ingreso real (Y) y la tasa de interés pasiva (I) se distribuyen individualmente como una normal según este test. 
Tabla. 1

Resumen de las estadísticas descriptivas de las variables del estudio

\begin{tabular}{llll}
\hline Descriptivos & LGC & LY & I \\
\hline Media & 15,98 & 16,43 & 5,17 \\
Mediana & 15,99 & 16,43 & 5,09 \\
Máximo & 16,24 & 16,71 & 9,40 \\
Mínimo & 15,54 & 16,03 & 3,58 \\
Desviación estándar & 0,20 & 0,22 & 1,03 \\
Asimetría & $-0,46$ & $-0,26$ & 1,74 \\
Curtosis & 1,97 & 1,72 & 7,24 \\
Jarque-Bera & 12,80 & 1,08 & 1,16 \\
Probabilidad & 0,00 & 0,58 & 0,56 \\
Observaciones & 76 & 76 & 76 \\
\hline \multicolumn{2}{c}{ Fuente: Elaboración propia }
\end{tabular}

Fuente: Elaboración propia

Como complemento al análisis individual de las series de tiempo del estudio, en la figura 2 se muestra la estimación de las tendencias y del ciclo de los logaritmos del gasto de consumo real (GC) y del ingreso real (Y) a través del filtro Hodrick-Prescott (HP) en donde se puede apreciar, en la parte inferior del gráfico, una relación medianamente estrecha entre el ciclo del gasto de consumo y del ingreso en términos reales (con un coeficiente de correlación simple de $0,65)$ lo cual refleja en cierto grado la respuesta del consumo del periodo corriente con respecto al nivel de ingreso del período corriente.

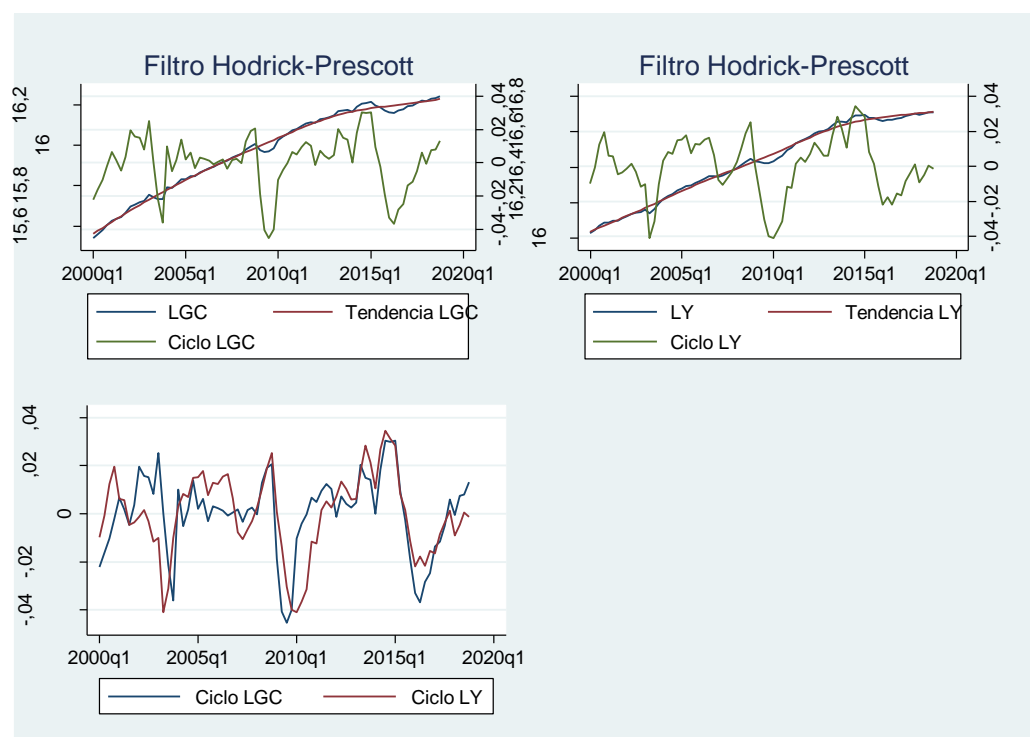

Figura 2. Comportamiento de la tendencia y del ciclo del gasto de consumo real y del ingreso real. Período (2000:q1-2018:q4).

Fuente: Elaboración propia

Continuando con el análisis previo de las variables, en los modelos de regresión que utilizan series de tiempo, es necesario evaluar la estacionariedad débil de las variables para los efectos de validar la inferencia estadísticas que pueden surgir de las estimaciones y evitar el 
problema de regresiones espurias (Gujarati y Porter, 2010). Para los efectos de este trabajo, se utilizaron las pruebas convencionales formales de raíces unitarias de Dickey-Fuller Aumentado (ADF) y Phillips-Perron (PP), además, de la inspección visual de las series y el gráfico del correlograma muestral para los casos que lo ameritaron (Gujarati y Porter, 2010).

En este sentido, en la tabla 2 se presentan los resultados de estas pruebas los cuales sugieren que para las tres variables, a saber LOG (GC), LOG (Y) e I, durante el período 2000:q1 2018:q4, las series son estacionarias en primeras diferencias, es decir son integradas de orden 1, I (1), en niveles, y al diferenciarlas se transforman en series estacionarias. Cabe señalar que para la serie de tasa de interés nominal pasiva en \% (I) la pruebas ADF y PP arrojaron resultados ambiguos por lo que el análisis se complementó con la inspección visual de las series y el gráfico del correlograma muestral los cuales arrojaron como resultados descartar la estacionariedad débil en niveles y concluir que las mismas son integradas de orden 1, I (1) al igual que el resto de las variables.

\section{Tabla. 2}

Pruebas de raíces unitarias para contrastar estacionariedad en las series de tiempo empleadas en el estudio.

\begin{tabular}{lcccccccc}
\hline Variable & \multicolumn{4}{c}{ Niveles } & \multicolumn{3}{c}{ Primeras diferencias } \\
& \multicolumn{2}{c}{ ADF1 } & \multicolumn{2}{c}{ PP1 } & \multicolumn{2}{c}{ ADF1 } & \multicolumn{2}{c}{ PP1 } \\
& CST & CCT & CST & CCT & CST & CCT & CST & CCT \\
\hline LOG (GC) & 0,030 & 0,749 & 0,0349 & 0,7306 & 0,000 & 0,000 & 0,000 & 0,000 \\
LOG(Y) & 0,106 & 0,989 & 0,2473 & 0,9617 & 0,000 & 0,000 & 0,000 & 0,000 \\
I & 0,000 & 0,002 & 0,0001 & 0,0051 & 0,000 & 0,000 & 0,000 & 0,000 \\
\hline
\end{tabular}

Notas: CCST: constante sin tendencia; CCCT: contante con tendencia; 1: Dickey-Fuller aumentado; 2: Philips Perron; 3: P-valores de acuerdo a Mackinnon (1996); decisión de acuerdo al test: Los residuos de la regresión son estacionarios en niveles y en primeras diferencias.

Fuente: Elaboración propia

\section{Resultados}

Una vez realizado el análisis previo de los datos, en este apartado se presentan las estimaciones econométricas realizadas para contrastar la hipótesis del ingreso permanente (TIP) en la estimación de la función de consumo trimestral para la economía ecuatoriana durante el período (2000:q1-2018:q4). Para dicho fin se estiman dos modelos sobre la base del modelo de racionalización de Koyck (1954) para la función de consumo siguiendo a Gujarati y Porter (2010), en este sentido, se estiman dos regresiones en niveles logarítmico, la primera, estimada por MCO y la segunda, utilizando el método de variables instrumentales (VI) específicamente el métodos de Mínimos Cuadrados en 2 Etapas (MC2E), verificando la estacionariedad de los residuos según las pruebas convencionales de raíz unitaria (ADF y PP) para los efectos de la inferencia estadística de los modelos. A continuación, se presentan las dos estimaciones con sus respectivas pruebas o análisis econométrico obtenido a través del programa Stata 13.0, para posteriormente comparar e interpretar los resultados de los parámetros relevantes.

En la tabla 3 se puede apreciar la estimación por MCO de la ecuación [15] en logaritmo la cual se expresa a continuación utilizando la notación de las variables del estudio: 


$$
\begin{gathered}
g c_{t}=\alpha(1-\lambda)+\beta_{0} y_{t}+\lambda g c_{t-1}+v_{t} \\
\text { Donde } v_{t}=\left(u_{t}-\lambda u_{t-1}\right) .
\end{gathered}
$$

Tabla. 3

Estimaciones del modelo de letargo geométrico de consume agregado para la economía de

\begin{tabular}{|c|c|c|c|}
\hline \multirow[b]{2}{*}{ Parámetros e indicadores } & \multirow[b]{2}{*}{ Variables } & \multicolumn{2}{|c|}{$\begin{array}{l}\text { Modelo en niveles (Logs) y expectativas adaptativas } \\
\text { (Racionalización del modelo de Koyck) }\end{array}$} \\
\hline & & $\begin{array}{l}\text { Consumo agregado } \\
\text { en Logs }\end{array}$ & $\begin{array}{l}\text { Consumo } \\
\text { agregado, } \\
\text { Método de VI } \\
\text { (MC2E) } \\
\text { en Logs } \\
\end{array}$ \\
\hline \multirow{2}{*}{$C$} & \multirow{2}{*}{ Constante } & 0,381 & 0,328 \\
\hline & & $(0,106)$ & $(0,082)$ \\
\hline \multirow{2}{*}{ 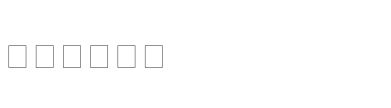 } & \multirow{2}{*}{ LOG(Y) } & 0,142 & 0,042 \\
\hline & & $(0,028)$ & $(0,046)$ \\
\hline \multirow{4}{*}{$\square$} & \multirow{2}{*}{$\operatorname{LOG}(\mathrm{GC}(-1))$} & $\mathbf{0 , 8 3 1}$ & 0,937 \\
\hline & & $(0,059)$ & $(0,043)$ \\
\hline & \multirow{2}{*}{ DUMMY } & $-0,013$ & \\
\hline & & $(-0,025)$ & \\
\hline \multicolumn{2}{|l|}{$\square \square \square \square \square$} & 0,169 & 0,063 \\
\hline \multicolumn{2}{|c|}{ 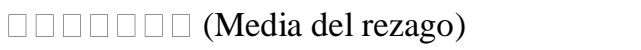 } & 3,013 & 4,917 \\
\hline \multicolumn{2}{|c|}{ Media del rezago $\square \square \log \square \log \square]$} & 2,419 & 3,744 \\
\hline \multicolumn{2}{|c|}{ Número de observaciones } & 75 & 74 \\
\hline \multicolumn{2}{|l|}{ R-cuadrado } & 0,997 & 0,997 \\
\hline \multicolumn{2}{|l|}{ R-cuadrado ajustado } & 0,997 & 0,997 \\
\hline \multicolumn{2}{|c|}{ Durbin-Watson } & 1,532 & 1,636 \\
\hline \multicolumn{2}{|c|}{$\begin{array}{l}\text { Prueba LM (Autocorrelación). Prob. Chi- } \\
\text { Cuadrado }\end{array}$} & 0,019 & 0,079 \\
\hline \multicolumn{2}{|c|}{$\begin{array}{l}\text { Prueba Hetoroscedasticidad. Prob. Chi- } \\
\text { Cuadrado }\end{array}$} & 0,506 & 0,295 \\
\hline \multicolumn{4}{|c|}{$\begin{array}{l}\text { Notas: }{ }^{1} \text { Variable instrumental (VI), específicamente el método de mínimos cuadrados en } 2 \text { etapas (MC2E). Las } \\
\text { variables instrumentales usadas son: una constante, el primer regresor, el primer letargo del regresor LOG (Y (- } \\
\text { 1)) y el segundo regresor en primer periodo de letargo LOG (GC (-1)). }\end{array}$} \\
\hline \multicolumn{4}{|c|}{$\begin{array}{l}\text { El LOG que precede la variable significa el logaritmo natural de dicha variable; una D (excepto en la variable } \\
\text { aleatoria) en frente de la variable significa la primera diferencia de la variable en mención, mientras cuando } \\
\text { DLOG precede una variable significa la primera diferencia del logaritmo natural de la misma variable (como una } \\
\text { aproximación de su tasa de crecimiento). }\end{array}$} \\
\hline \multirow{2}{*}{\multicolumn{4}{|c|}{$\begin{array}{l}\text { Los residuos de los modelos estimados con las variables en niveles fueron estacionarios, I (0), de acuerdo a las } \\
\text { pruebas ADF y PP. } \\
\text { Los modelos fueron estructuralmente estables en los parámetros de acuerdo de las pruebas Cusum y Cusum } \\
\text { cuadrado. } \\
\text { Los coeficientes son significativos al 5\%. Los errores estándar entre paréntesis (). }\end{array}$}} \\
\hline & & & \\
\hline \multicolumn{4}{|c|}{$\begin{array}{l}\text { La variable denotada como DUMMY es una variable aleatoria que toma el valor de uno (1) en el primer cuarto } \\
\text { del año } 2009 \text { (2009:q1) y cero (0) en el resto del periodo. } \\
\text { Fuente: Elaboración propia }\end{array}$} \\
\hline
\end{tabular}
Ecuador. Periodo (2000:q1-2018:q4) 
Esta función de consumo se denomina la función de consumo de corto plazo. En donde todos sus parámetros a nivel individual y en conjunto son significativos. Para derivar la función de consumo de largo plazo se parte de los resultados del cuadro $3^{6}$, en donde se puede apreciar que $\lambda=0.831$ y $\beta_{0}=0.142$. Entonces $\beta_{1}=(0.142)(0.831) \approx 0.118, \beta_{2}=$ $(0.1214)(0.751)^{2} \approx 0.098$ y así sucesivamente, que son los multiplicadores de corto y mediano plazos. Finalmente, con la ecuación [10] obtenemos el multiplicador de largo plazo, es decir, el efecto total del cambio del ingreso sobre el consumo después de tomar en cuenta todos los efectos rezagados, que en el presente caso es:

$$
\sum_{k=0}^{\infty} \beta_{k}=\beta_{0}\left(\frac{1}{1-\lambda}\right)=(0.142)\left(\frac{1}{1-0.831}\right)=0.838
$$

En otras palabras, un incremento sostenido de un dólar en el $y_{t}$ producirá al final alrededor de 0.84 dólares de aumento en el $g c_{t}$, pero el impacto inmediato, o de corto plazo, es de sólo 0.14 dólares. Además, como se puede observar en la parte inferior de la Tabla 3, los supuestos de no-autocorrelación y heterocedasticidad fueron respetados.

$\mathrm{Al}$ igual que en el caso anterior (MCO) para derivar la función de consumo de largo plazo se parte de los resultados del cuadro 4 , en donde se puede apreciar que $\lambda=0.937$ y $\beta_{0} \approx 0.042$. Por lo tanto $\beta_{1}=(0.042)(0.937) \approx 0.039, \beta_{2}=(0.042)(0.937)^{2} \approx 0.037$ y así sucesivamente, que son los multiplicadores de corto y mediano plazos. Por último, tal como en el caso anterior, con la ecuación [10] obtenemos el multiplicador de largo plazo, es decir, el efecto total del cambio del ingreso sobre el consumo después de tomar en cuenta todos los efectos rezagados, que en el presente caso es:

$$
\sum_{k=0}^{\infty} \beta_{k}=\beta_{0}\left(\frac{1}{1-\lambda}\right)=(0.042)\left(\frac{1}{1-0.937}\right)=0.666
$$

En otras palabras, un incremento sostenido de un dólar en el $y_{t}$ producirá al final alrededor de 0.66 dólares de aumento en el $g c_{t}$, pero el impacto inmediato, o de corto plazo, es de sólo 0.04 dólares, valores muy similares a los reportados por MCO (0.84 y 0.14, respectivamente).

De las dos estimaciones realizadas, como se muestra en el cuadro 3, la evidencia disponible durante el período de estudio sugiere que el coeficiente de expectativas $(1-\lambda)$ se encuentra entre un rango de 0,06 a 0,17 , tomando en consideración las ventajas y desventajas relativas de cada una de las especificaciones, lo cual arroja indicios, de que sobre la base del modelo de expectativas adaptativas, el $g c$ real se ajusta al $y$ con un rezago considerable. La teoría señala que cuanto mayor sea el valor de $\lambda$, que toma valores entre cero y uno, mayor será el tiempo que tardará en reflejar el impacto de un cambio en el valor de la variable explicativa en la variable dependiente. Lo cual arroja evidencia a favor de la TIP, en el sentido de que el gasto

\footnotetext{
${ }^{6}$ Se incluyó una variable dummy para el primer trimestre del año 2009 (dummy09) para recoger algunos eventos exógenos que afectaron a la economía ecuatoriana en el año 2009, por la caída de los precios internacionales del petróleo, entre otros factores.
} 
de consumo de los hogares responde más a los cambios en el ingreso permanente que al ingreso transitorio.

\section{Conclusiones}

Unos de los temas más estudiados empíricamente en la macroeconomía es el consumo, tanto por sus implicaciones en las fluctuaciones económicas de corto plazo (en la demanda agregada), como por sus efectos a largo plazo entre la distribución de los recursos entre el consumo y el ahorro, factor clave del crecimiento económico. En este trabajo, se realizó una breve revisión de la literatura sobre la hipótesis del ingreso permanente (Friedman, 1957) para que sobre la base de los postulados de esta hipótesis y del modelo de Koyck (1954) estimar una función de consumo para la economía de Ecuador con datos de frecuencia trimestral durante el período (2000:q1-2018:q4), con el propósito de contrastar, en cierta forma, la hipótesis o TIP.

Para intentar lograr dicho objetivo, se estimaron dos regresiones a través de dos métodos de estimación, a saber, MCO y MC2E, utilizando principalmente la estimación de una racionalización del modelo de Koyck (Gujarati y Porter, 2010) para el consumo agregado. Los resultados de estas estimaciones sugieren, según la base de la evidencia disponible, que el coeficiente de expectativas $(1-\lambda)$ se encuentra entre un rango de 0,06 a 0,17 ; tomando en consideración las ventajas y desventajas relativas de cada una de las especificaciones, lo cual arroja indicios, de que al parecer, el $g c$ real se ajusta al nivel de ingreso real $(y)$ con un rezago considerable: tomando en cuenta que cuanto mayor sea el valor de $\lambda$ (entre 0 y 1 ), más tiempo tardará en sentirse el impacto pleno de un cambio en el valor de la variable explicativa en la variable dependiente. Lo cual arroja evidencia a favor de la TIP, en el sentido de que el gasto de consumo de los hogares responde más a los cambios en el ingreso permanente que al ingreso transitorio.

\section{Bibliografía}

Aguiar, M., \& Pence, K. (2014). Comments and Discussion. Brookings Papers on Economic Activity, pp. 139-153. Retrieved from http://www.jstor.org/stable/23936273

Argandoña, A. (1990). El pensamiento económico de Milton Friedman (3). Recuperado de http://www.iese.edu/research/pdfs/DI-0193.pdf

Banco Central del Ecuador. (2017). BCE Información Económica. Recuperado de https://www.bce.fin.ec/index.php/informacioneconomica

De Gregorio, J. (2007). Macroeconomía: teoría y política. México: Pearson Educación.

Duesenberry, J. (1967). Income, saving, and the theory of consumer behavior (180). United States: Oxford University Press.

Fisher, I. (1930). The theory of Interest. United States: Macmillan Co.

Friedman, M. (1957). A theory of the Consumption Function. United States: Princeton University Press.

Greene, W. (1999). Análisis econométrico (Ed. 3). Madrid: Prentice Hall.

Gujarati, D. \& Porter, D. (2010). Econometría (Ed. 5). México: Mc Graw Hill.

Instituto Nacional de Estadísticas y Censos. (2017). INEC Estadísticas. Recuperado de https://www.ecuadorencifras.gob.ec/estadisticas/

Keynes, M. (1936). The General Theory of Employment, Interest, and Money. London: Macmillan.

Esta obra se comparte bajo la licencia Creative Common Atribución-No Comercial 4.0 International (CC BY-NC 4.0)

Revista de la Universidad Internacional del Ecuador. URL: https://www.uide.edu.ec/ 
Koyck, L. (1954). Distributed Lags and Investment Analysis. Amsterdam: North Holland Publishing Company.

Liquitaya, J. (2014). La teoría del ingreso permanente: Análisis empírico de 19 países latinoamericanos. Denarius, pp. 43-64.

Mankiw, G. (2014). Macroeconomía (Ed. 8). Barcelona, España: Antoni Bosch.

Modigliani, F. \& Brumberg, R. (1954), Utility analysis and the consumption function: An interpretation of cross-section data in Kenneth K. Kurihara. Post-Keynesian Economics: New Brunswick, N.J., pp 388-436.

Romer, D. (2006). Macroeconomía avanzada (Ed. 3). Madrid: Mc Graw - Hill.

Yang, Y. (1964). An international comparison of consumption functions. The Review of Economics and Statistics, pp. 279-286. 